\title{
Molecular Spectroscopy in Astrophysics: The case of Polycyclic Aromatic Hydrocarbons
}

\author{
Farid Salama \\ NASA-Ames Research Center, Space Science Division, \\ Mail Stop 245-6, Moffett Field, California 94035-1000, USA \\ fsalama@mail.arc.nasa.gov
}

October 6,2000

\begin{abstract}
The role of molecular spectroscopy in astrophysics and astrochemistry is discussed in the context of the study of large, complex, carbonbearing molecules, namely, Polycyclic Aromatic Hydrocarbons or PAHs. These molecular species are now thought to be widespread in the interstellar medium in their neutral and ionized forms. Identifying the carriers responsible for uniclentified interstellar spectral bands will allow to derive important information on cosmic elemental abundances as well as information on the physical conditions (density, temperature) reigning in specific interstellar environments. These, in turn, are key elements for a correct understanding of the energetic mechanisms that govern the origin and the evolution of the interstellar medium. A multidisciplinary approach -combining astronomical observations with laboratory simulations and theoretical modeling-is required to address these complex issues. Laboratory spectra of several PAHs, isolated at low temperature in inert gas matrices or seeded in a supersonic jet expansion, are discussed here and compared to the astronomical spectra of reddened, early type, stars. The electronic spectroscopy of PAHs in the ultraviolet, visible and near-infrared domains is reviewed and an assessment of the potential contribution of PAHs to the interstellar extinction in the ultraviolet and in the visible is discussed.
\end{abstract}

Keywords: PAHs, Electronic, Spectroscopy, Diffuse Interstellar Medium 


\section{Introduction}

Molecular spectroscopy plays an important role in astrophysics and in astrochemistry. This is because photons carry most of the information that we are able to remotely access regarding the nature and the composition of stellar and interstellar objects that lay far away from Earth. Astrophysics covers a wide range of issues ranging from the study of our solar system to the study of the interstellar medium (ISM). The objectives of laboratory astroplysical studies are to simulate as closely as possible the conditions known or expected to exist in a given space environment and to provide quantitative data which are relevant for the interpretation of astronomical observations. The role of laboratory astrophysics is thus essential for testing and improving the existing astrophysical models and for providing an accurate understanding of astronomical observations (for recent reviews on this topic see [31]; [21] for solar system studies and [20], [22] for interstellar studies). Laboratory astrophysical studies are illustrated, here, through the case of the diffuse interstellar absorption bands (DIBs). DIBs are a set of ubiquitous absorption features which remains unidentified since its discovery by Heger in 1922. The DIB spectrum consists of more than two hundred confirmed interstellar bands including a large number of weak features (For a. recent review, sce [10]; see also Sarre in the same issue). Here, we discuss the potential link between the polycyclic aromatic hydrocarbon (PAH) ions and the carriers of the bands (for an in-depth discussion see [20], [22]). The PAH-DIB proposal has been put forward, more than a decade ago, on the basis of the expected abundance of PAHs in the interstellar medium and their stability against UV photodissociation [34], [14], [4]. PAHs are now thought to be largely responsible for the discrete infrared emission bands observed at $3.3,6.2,7.7,8.6$ and $11.3 \mu \mathrm{m}$ in many astronomical objects including HII regions, planetary and reflection nebulae, and the interstellar medium of the Milky Way and other galaxies [2]. Recent observations from the IR space satellites ISO [16] and IRTS [17] have confirmed that PAHs are ubiquitous throughout the general diffuse interstellar medium as well. According to the astrophysical model, PAHs are expected to be present as a mixture of free. neutral and ionized, molecules following a large size distribution which range from small, gas-phase, molecules ( $\leq 25$ carbon atoms) to large graphitic platelets [1], [18]. PAHs are considered to form a link between the gas and the solid phase of interstellar dust and to be a key element for the coupling of stellar FUV photons with the interstellar gas. 
The PAII-DIB proposal has been tested in the laboratory by measuring the spectra of neutral and ionized PAHs taken under astrophysically relevant conditions. In the laboratory experiments, PAHs have traditionally been isolated at very low temperature (5 degree Kelvin) in inert gas (neon) solid matrices [23], [24], [25], [26], [27], [5], [6], [15]. Recent developments in the detection techniques have allowed to measure, for the first time, the spectra of these large molecules and ions in the gas phase either through direct absorption with cavity ring down spectroscopy, CRDS [19] or using molecular depletion techniques [3]. The UV to NIR wavelength range is key element to the potential unambiguous identification of specific PAHs in the interstellar medium because of the unique vibronic spectral signature of molecules and ions (as opposed to the IR transitions which are only characteristic of molecular functional side groups). Based on these laboratory data and on the available astronomical data, the PAH-DIB proposal has been discussed [20], [28], [29] with the conclusion that PAH ions are indeed very promising candidates for the DIB carriers. A review of these findings is presented in the following sections.

\section{Diffuse interstellar bands}

The diffuse interstellar bands (DIBs) are absorption features superposed on the interstellar extinction curve. DIBs have their origin in the diffuse interstellar medium which is one of the various phases present in the interstellar medium (ISM). The diffuse interstellar medium is characterized by low density $\left(25-30 \mathrm{~cm}^{-3}\right)$, cold $(\mathrm{T}<100 \mathrm{~K}$ ) clouds of dust and gas (for a review see [32]). DIBs fall in the near ultraviolet to the near-infrared range (4400 - $10,000 \AA$ ). The bands are characterized as diffuse because they are broad and shallow in comparison to the well-known, narrow, interstellar atomic lines. The individual DIBs vary widely in strength and shape, with equivalent width per magnitude of visual extinction ranging from about $2 \AA$ to the detection limit of about $0.006 \AA$. The full width at half maximum (FWHM) values for the DIBs range from about 0.4 to $40 \AA$

The identification of the carriers responsible for these features remains elusive. Various candiclates have been proposed as possible carriers for the bands, ranging from impurity-doped dust grains, to free, neutral and ionized, molecular species of varying sizes and structures to molecular hydrogen [33]. It is generally admitted today, however, that the concept of a single carrier 
must be disiegarded due to the large number of bands detected and the lack of correlation between the bands. It is now thought that the carriers are most probably gas-phase molccules in either the neutral and/or ionized forms and that these molecular carriers are part of an extended size distribution of the interstellar dust. Structure within the profiles of a few strong DIBs is now seen in high-resolution observations ([30], [7], [12], [13]). This structure is generally interpreted as the signature of the rotational band structure of gas-phase molecules and supports an origin of the DIBs in C-rich molecules.

Identifying the DIB carriers, which are a significant constituent of the interstellar medium, is crucial to a correct understanding of the heating and cooling mechanisms governing the origin and evolution of the interstellar medium. The cooling of the diffuse clouds through atomic/molecular line emission is balanced by the heating of the gas by cosmic rays and photons. This latter mechanism is dominated by the coupling of FUV photons ( $h \nu \geq$ $13.6 \mathrm{eV}$ ) to the gas predominantly through large (polyatomic) molecules and small grains (up) to $15 \AA$ size).

\section{Comparison of the laboratory spectra with as- tronomical observations}

The astrochemical study, described below, is based on the comparison between observational and laboratory data. Heavily reddened stars have been selected to facilitate the detection of weak spectral features. The wavelength coverage ranges $f_{100 \mathrm{~m}} \sim 3500 \AA$ to $\sim 10,100 \AA$ with a resolution ranging from 45,000 to 60,000 . The details of the observational procedure are described in [29].

The experimental studies have been described in [22] and only a synopsis is given here. A proper simulation in the laboratory of the experimental conditions reigning in the diffuse interstellar medium requires to combine cryogenic techniques with high-vacuum techniques and molecular samples in the gas phase. Matrix isolation spectroscopy (MIS) is used, in a first approximation, to simulate in the laboratory the environmental conditions which are close to the conditions known (or expected) in the diffuse interstellar medium. In MIS experiments, the neutral and ionized PAHs are isolated at low temperature $(<5 \mathrm{~K})$ in neon matrices where the perturbations induced in the spectrum of the trapped molecules and ions are minimum [20]. Until PAHs can be routinely studied in supersonic jets, MIS remains the best tool 
arailable to simulate the conditions of the diffuse interstellar medium. The experimental apparatus used for MIS studies [26] consists of a high-vacuum, cryogenic, sample chamber equipped with 4 ports, two gas injection ports and a sample holder suspended at the center of the chamber. The sample holder is cooled down to $5 \mathrm{IS}$ by a liquid He transfer cryostat. The spectrometer is composed of a triple grating monochromator and a CCD detector and covers the wavelength range $180-1100 \mathrm{~nm}$. The ionization field is provided by a microwave- powered, flow discharge hydrogen lamp generating photons of $10.2 \mathrm{eV}$ energy (Jyman a line). The PAH sample is simultaneously condensed with the neon gas onto the cold substrate. The frozen matrix is then spectroscopically analyzed. Ions are generated in situ from the stable precursor, via vacuum-ultraviolet (VUV) photoionization. VUV irradiation of the neutral P.Hs isolated in Ne matrices produces new spectral features in the UV-NIR range $(1800-10,600 \mathrm{~nm})$. The new features are found to be associated with the PAH cation $\left(P A H^{+}\right)$formed by direct, one- photon, ionization of the neutral precursor. Some representative spectra are shown in Figures 1 to 4 for the PAH cations phenanthrene $\left(C_{14} H_{10}^{+}\right)$, benzo(e)pyrene $\left(C_{20} H_{12}^{+}\right)$. benzo(ghi)perylene $\left(C_{22} H_{12}^{+}\right)$and pentacene $\left(C_{22} H_{14}^{+}\right)$.

The astrophysical implications derived for neutral and ionized PAH have been discussed in the literature $([28],[20],[29])$ and are recapitulated below: (i) The absorption spectra of all small ( $\leq 25 \mathrm{C}$-atoms), neutral PAHs exhibit a series of discrete transitions in the UV-NUV range $(180-400 \mathrm{~nm})$ with no absorption in the visible-NIR range. The absorption band energies of neutral P:AHs shift, towards lower energies (longer wavelengths) when the molecular size increases. The absorption band systems are associated with vibronic transitions between the electronic states of the molecule and can be classified, in a first approximation, into very strong (oscillator strength, $f$, of the order of 1 ), moderately strong ( $f$ of the order of 0.1 ) and weak ( $f$ of the order of 0.001 ).

(ii) Contrary to thcir neutral precursors, ionized PAHs absorb in the visible and NIR and could contribute to the DIBs (see Figures 1 to 4). Large PAH ions ( $\geq 100$ rings, i.e., containing more than 250-300 C-atoms) are not expectcd, however, to contribute significantly to the DIBs because their strongest absorption falls outside the wavelength range in which the DIBs are observed ([28], [20]).

(iii) The absorption spectrum of PAH cations is dominated by a single band in the spectral range of interest for comparison with the DIBs (i.e., NUV. NIR; [29]). 
(iv) In the case of compact PAHs (such as $\mathrm{C}_{20} \mathrm{H}_{12}^{+}$and $\mathrm{C}_{22} \mathrm{H}_{12}^{+}$shown in Figures 1 and 2), the strongest absorption lies at the high energy end of the spectrum and its oscillator strength is of the order of 0.1 . In the case of non-compact PAHs (such as $\mathrm{C}_{10} \mathrm{H}_{8}^{+}, \mathrm{C}_{14} \mathrm{H}_{10}^{+}$and $\mathrm{C}_{22} \mathrm{H}_{14}^{+}$shown in Figures 3 and 4 ), the strongest absorption lies at the low energy end of the spectrum and has an oscillator strength of the order of 0.1 .

In summary, PAII ions produce one strong band (typical oscillator strength of 0.1 ) together with several weaker features in the NUV-to-NIR range.

When comparing the spectra of heavily reddened stars (Figs 5 - 6) in the vicinity of the major PAH ion features measured in laboratory experiments, features were searched for in 100 Angstrom windows around laboratory peak wavelength positions [29]. The window for comparison covers thus a fractional shift of $0.5 \%$ in energy for the laboratory measured absorption bands in agreement with the expected shift induced by the solid matrix environment [19]. [20]. The findings resulting from such a comparison are reviewed in the following section.

\section{Discussion}

DIBs can be roughly classified as strong (band equivalent widths, $W_{\lambda}, \geq 1.0$ $\AA)$, moderately strong $\left(1.0 \AA \geq W_{\lambda} \geq 0.1 \AA\right)$, and weak $\left(W_{\lambda} \leq 0.1 \AA\right)$ [28]. There are only 3 strong DIBs and less than 50 moderately strong DIBs tabulated in [10] towards the star IID 183143. All the rest of the DIBs (i.e., the rast majority) range from weak to very weak (less than $0.01 \AA$ equivalent widths). All the weak features exhibit the same characteristics as the well-known, sl.ronger, DIBs ([7], [12]. [11], [13]) and are also most likely of molecular origin. These features are observed in practically all environments, including the very diffuse clouds [8]. This indicates that the (molecular) carriers of the weak DIBs must be resistant against the UV photon background.

As illustrated in Figures to 4, the spectrum of a PAH cation in the NUY-to-NIR Spectral range is characterized by a single, strong feature and several related woak bands (i.e. weaker by, at least, an order of magnitude). The weaker bands associated with a specific PAH ion are expected to be observed only when the strongest PAII feature is associated with one of the strong or moderatcly strong, well-known, DIBs. Inversely, if the strongest 
feature of a PAII ion corresponds to one of the weak DIBs observed in our spectra (i.c., the features observed at the level of detection) there is, obviously, no possibility of detecting the weaker PAH band relatives which would be no decper than 0.001 of the continuum. In other words, there are two scenarii which can be met while attempting to identify specific DIBs with specific PAII ions: (i) a situation where many absorption bands of a single PAH ion correlate with a combination of strong and weak DIBs. This is the most rewarding situation where a decisive and unambiguous spectral identification can be made based on the comparison of the wavelength positions, energy intervals and relative intensities of numerous bands, (ii) a situation where the strongest absorption band of a PAH ion correlates with a weak DIB. This in itself does not represent a spectral fit. The assignment gains credibility, however, if a significant number of PAH ions (10 or more) are found to be correlated with weak DlBs. Examining the laboratory (MIS) spectra of PAH ions against these two criteria it has become possible to identify a number of PAH ions which could be potential DIB carriers [29]. A third of the elcments in the laboratory database exhibit a positive correlation with DIBs. Because of the small neon matrix-to-gas phase shift, the selected PAH ions must then be studied in the gas phase in jet expansions to provide the test for a decisive comparison in wavelengths and band profiles with the astronomical spectra. Moreover, out of the 24 DIBs which are found to fall in the wavelength windows, 7 can be described as strong to moderatcly strong while the rest of the bands range from weak to very weak.

In conclusion, it has been found that the direct comparison of the MIS spectra of PNH ions with the spectra of selected reddened stars cannot yield to a decisive, unambiguous, identification of DIB carriers. This is because (i) the spectra measured in the laboratory are subject to band position shifts induced by the solid inatrix and (ii) the stellar spectra contain so many interstellar features that only a precise experimental determination of the wavelengths of molecular features together with their gas-phase profile can allow a definitive identification. A comparison between the MIS laboratory data and the astronomical observations is useful, however, when the matrixto-gas-phase shift is taken into account. The MIS data provide, then, an essential guide for the selection of P:HH ions to be spectroscopically studied in a jet cxpansion (i.e., under conditions which mimic the conditions reigning in the interstellar medium) much more challenging experimentally. The MIS data indicate that a substantial number of PAH ions are promising canclidates for the DIB carriers. The jet experiments which are just 
now being devcloped in our laboratory will provide the much needed data to definitively asscss the validity of the PAH proposal with regards to the DIBs. More astronomical survcys of J)IB objects are also needed, especially in the wavelength ranges which have not been as yet frequently observed.

Substantial progress has been made in astrochemistry in recent years in terms of the amount and the quality of the laboratory and observational data available to address important astrophysical and astrochemical issues. Solving open questions such as the identity of the carriers of the DIBs, the origin of the unidentified infrared emission bands, the nature of the materials leading to the observed interstellar extinction in the ultraviolet now seems to be within reach. On the laboratory front, the limits of the current exporimental approach (i.e., MIS in our case) have been reached. New techniques in low temperature spectroscopy are now being developed or adapted to address these important problems. The combination of a free jet expansion with a cavity ring down [19] appears to be the most promising approach.

\section{Acknowledgements}

This work has been supported by grants from NASA (Office of Space Science, Astrophysics program).

\section{References}

[1] Allamandola, L. J., Tielens, A. G. G. M. and Barker, J. R. 1989, Astrophys. J. Sup, 71, 733

[2] Allamandola, L. J., Hudgins, D. M. and Sandford, S. A. 1999, Astrophys. .J., 511, L115

[3] Bróchignac, Ph., and Pino, Th. 1999, Astron. Astrophys., 343, 49

[1] Crawford, M. K., Tielens, A. G. G. M., and Allamandola, L. J. 1985, Astrophys. J., 293, L45

[5] Ehrenfreund, P., d'Hendecourt, L., Verstraete, L., Léger, A., Schmidt. W. and Defournean, D. 1992, Astron. Astrophys., 259, 257 
[6] Ehrenfreund, P., Foing, B. Il., d'Hendecourt, L., Jenniskens, P. and Désert. F. X. 1995, Astron. Astrophys., 299, 213

[7] Ehrenfreund, P. and Foing, B. II. 1996, Astron. Astrophys., 307, L25

[8] Cialazutdinov, G., Krelowsli. .J., Moutou, C. and Musaev, F. 1998, Mon. Not. Roy. Astron. Soc. 295, 437

[9] Heger. M. L. 1922, Lick Obs. Bull., 10, 146

[10] Herbig, G. H. 1995, Ann. Rer. Astron. Astrophys., 33, 19

[11] Jenniskens, P. and Dèsert, X. 1994, Astron. Astrophys. Sup, 160, 39

[12] Jenniskens, P., Porccddu, I., Benvenuti, P., and Dèsert, X. 1996, Astron. Astrophys. Sup. 313, 619

[13] Kirr. T. H., Hibbins, R. E., Fossey, S. J., Miles, J. R. and Sarre, P. J. 1998, Astrophys. J., 495, 9.11

[14] Léger, A., and d'Hendecourt, L. B. 1985, Astron. Astrophys., 146, 81

[15] Léger. A.. d'Hendecourt, I. and Défourneau, D. 1995, Astron. Astroplys., 293, 53

[16] Mattila, Ki et al. 1996, Astron. Astrophys. 315, L353

[17] Onaka, T. et al. 1996, Publ. Astron. Soc. Japan 48, L59

[18] Puget. J. L. and Léger, A. 19\$9, Ann. Rev. Astron. Astrophys., 27, 161

[19] Romanini, D., Biennier, L., Salama, F., Katchanov, A., Allamandola, L. J.. Stoeckel, F. 1999, Chem. Phys. Lett., 303, 165

[20] Salama, F. 1996, in Low Temperature Molecular Spectroscopy, R. Fausto ed., Kluwer, 483, 160

[21] Salama, F. 1998, in The Solur System Ices B. Schmitt, C. de Bergh, and M. Festou eds., Astrophysics and Space Science Library Series, Kiluwer Academic Publishers, p. 259

[22] Salama, F. 1999. In Solid Intcrstellar Matter: The ISO Revolution, L. d'Hendecourt, C. Joblin and A. Jones eds., EDP Sciences, SpringerVerlag. Les Ulis, p. 65 
[23] Salama, F.. and Allamandola. I.. J. 1991, J. Chem. Phys., 94, 6964

[24] Salama, F., and Allamandola, I.. J. 1992a, Astrophys. J., 395, 301

[25] Salama, F., and Nllamandola, I. J. 1992b, Nature 358, 42

[26] Salama, F., Joblin, C., and Allamandola, L. J. 1994, J. Chem. Phys., 101. 10252

[27] Salama, F., Joblin, C'., and Nllamandola, L. J. 1995, Planet. Space Sci. $+3,1165$

[28] Salama, F., Bakes, E. L. O.. Nlamandola, L. J. and Tielens, A. G. G. M. 1996, Astrophys. J., 458, 621

[29] Salama, F., Galazutdinov, G. A., Krelowski, J., Allamandola, L.J., and. Musaev, F.A. 1999, Astrophys. J., 526, 265

[30] Sarre, P., Miles, J. R.. Kerr, T. H., Hibbins, R. E., Fossey, S. J., and Somerville, W. B. 199.5, Mon. Not. Roy. Astron. Soc., 277, L41

[31] Smith, P. L., and Wiese, W. I. 1992, eds., Atomic and molecular data for Space Astronomy. Needs. Inalysis, and Availability, SpringerVerlag Berlin Heidelberg

[32] Tielens, A. G. G. M. 1995, in . lirborne Astronomy Symposium on the Galactic Ecosystem: From Gas to Stars to Dust, M. R. Haas, J. A. Daviclson, 'and E. F. Erickson eds., ASP Conference Series, Vol. 73, p.3

[33] Tielens, A. G. G. M. and Snow, T. P. (eds) 1995, The Diffuse Interstcllar Bands, IAU Coll. 137. Kluwer, Dordrecht

[34] Van dor Zwet, G. P., and Allımandola, L. J. 1985, Astron. Astrophis., $116,76$. 


\section{Figure captions}

Figure 1: The visible-NIR absorption spectrum of the compact PAH cation, benzo(e)perylene $\left(\mathrm{C}_{20} \mathrm{H}_{12}^{+}\right)$, isolated in a neon matrix at $5 \mathrm{~K}$ (From [22]).

Figure 2: The visible-NIR absorption spectrum of the compact PAH cation, benzo(ghi)poryene $\left(\mathrm{C}_{22} \mathrm{H}_{12}^{+}\right)$, isolated in a neon matrix at $5 \mathrm{~K}$ (From [22]).

Figure 3: The visible-NIR absorption spectrum of the non-compact PAH cation, phenanthrene $\left(\mathrm{C}_{14} \mathrm{H}_{10}^{+}\right)$, isolated in a neon matrix at $5 \mathrm{~K}$ (From [22]).

Figure 4: The visible-NIR absorption spectrum of the non-compact $\mathrm{PAH}$ cation, pentacene $\left(\mathrm{C}_{22} \mathrm{H}_{14}^{+}\right)$, isolated in a neon matrix at $5 \mathrm{~K}$ (From [22]).

Figure 5: A sample of DIBs is shown in the 6700 Aregion for 3 reddened stars (From [29]).

Figure 6: A sample of DIBs is shown in the 6500 A region for 3 reddened stars (From [29]). 


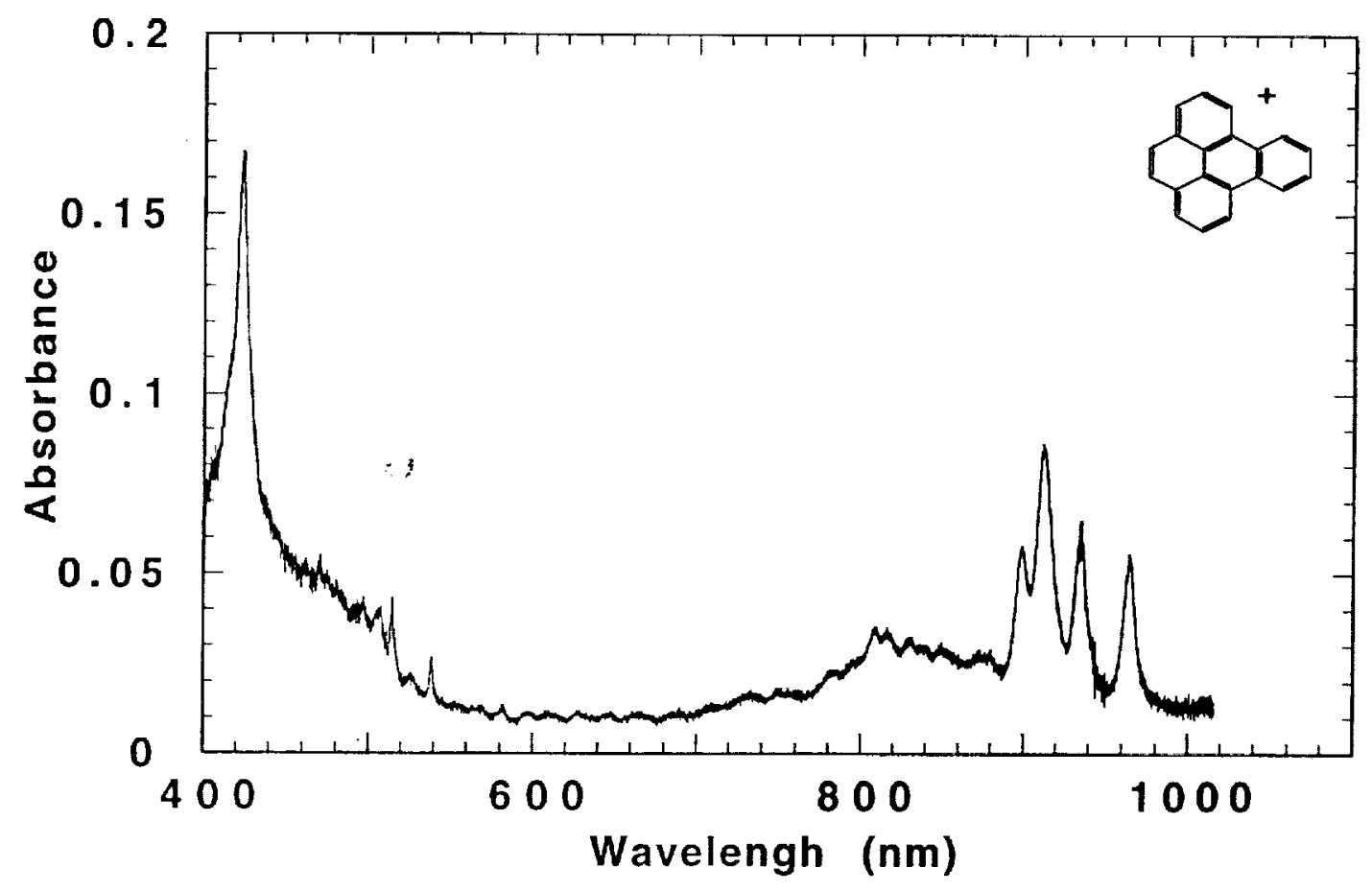




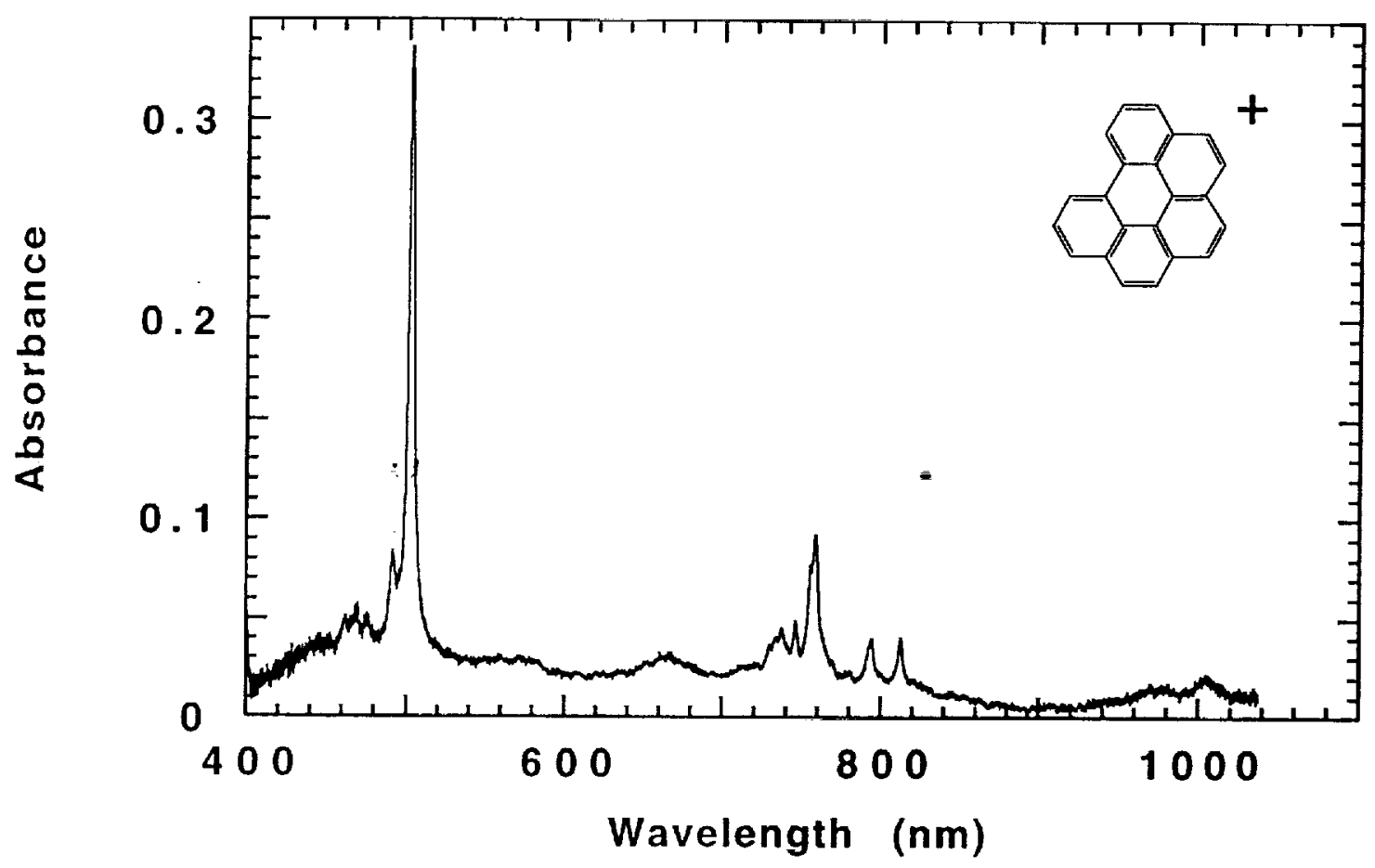




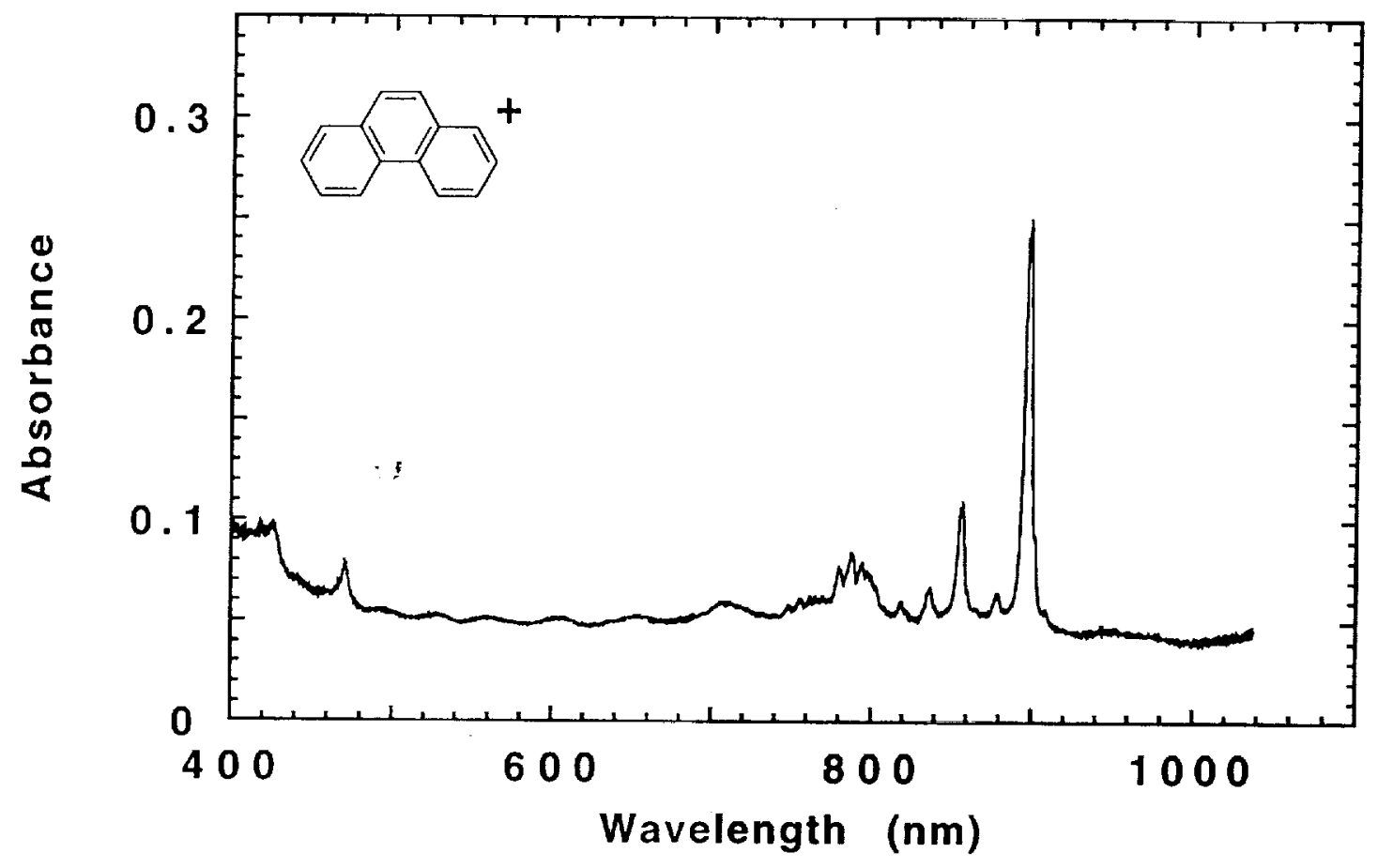




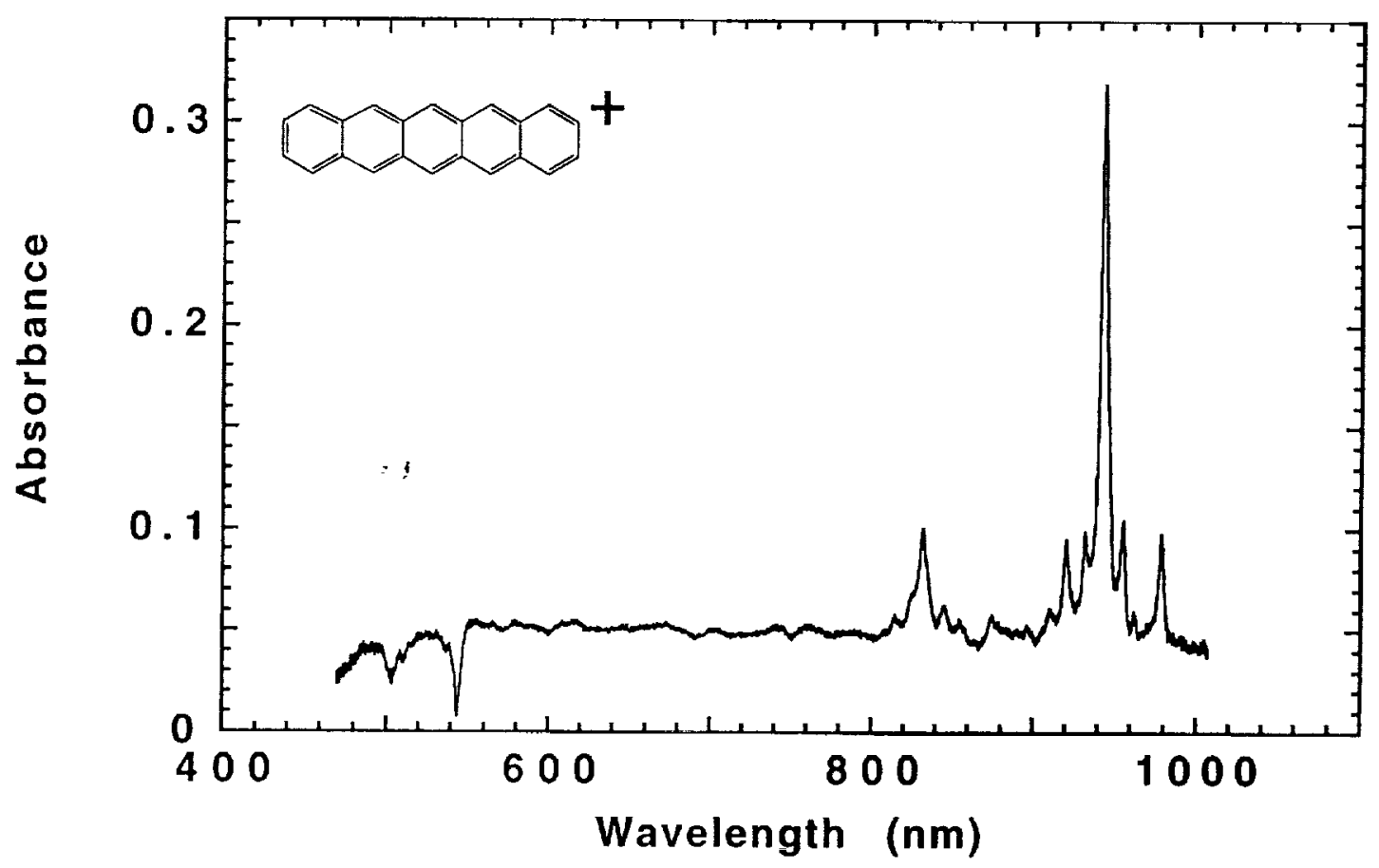




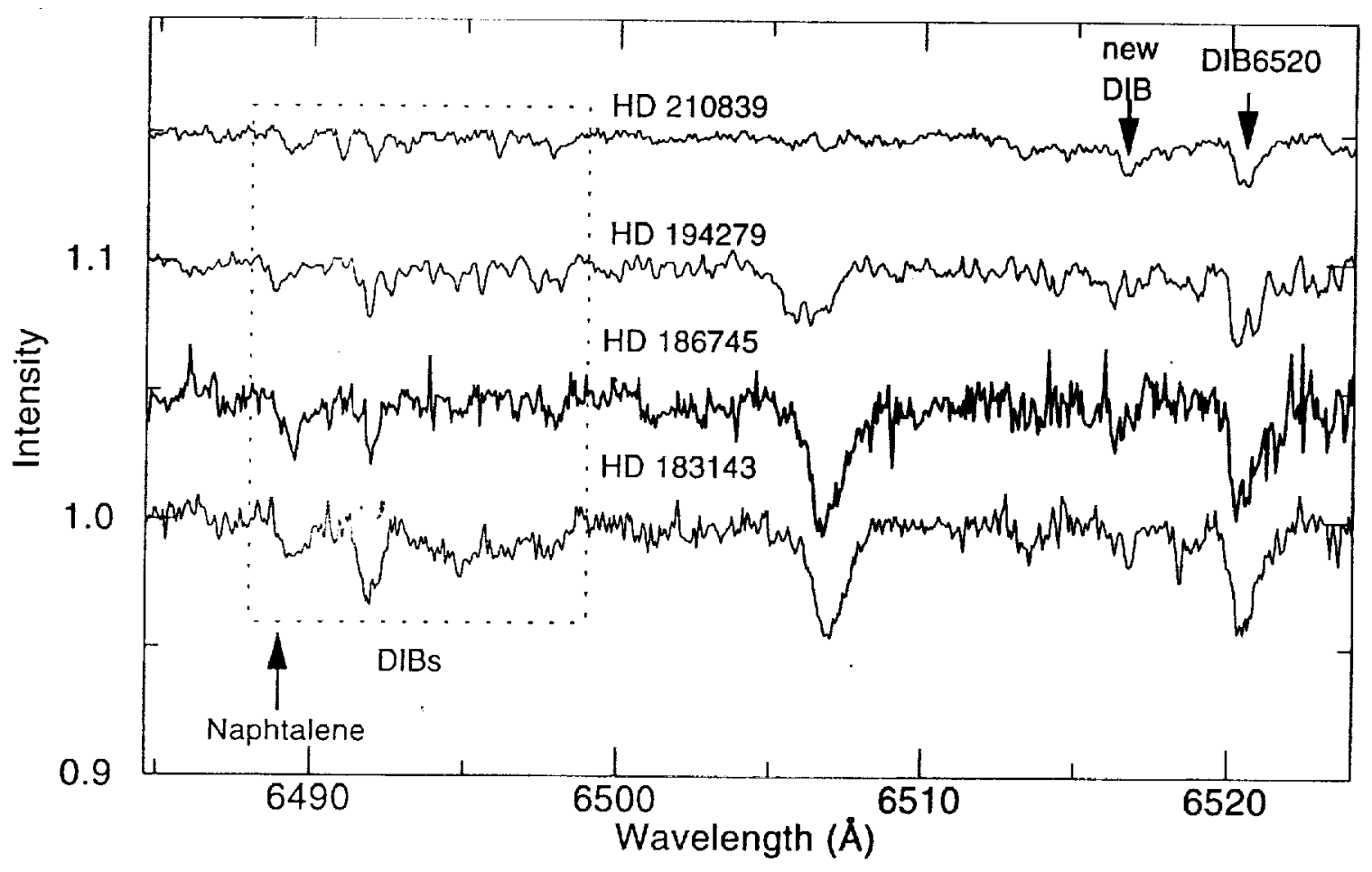




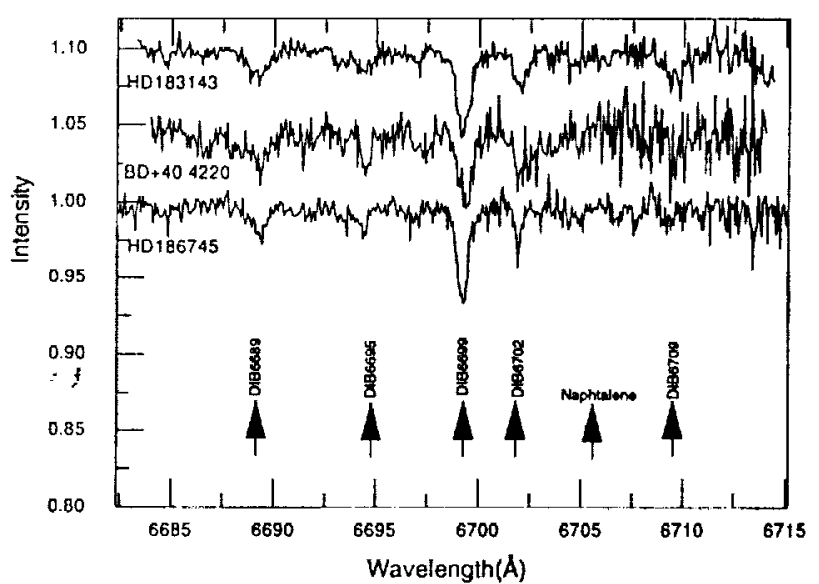

